\title{
Variables of the insulin resistance syndrome are associated with reduced arterial distensibility in healthy non-diabetic middle-aged women
}

\author{
N.M. van Popele ${ }^{1}$, I. C.D. Westendorp ${ }^{1}$, M.L. Bots ${ }^{2}$, R.S. Reneman ${ }^{3}$, A.P. G. Hoeks ${ }^{4}$, A.Hofman ${ }^{1}$, D. E. Grobbee Hof $^{2}$ \\ J.C.M. Witteman ${ }^{1}$ \\ ${ }^{1}$ Department of Epidemiology and Biostatistics, Erasmus University Medical School, Rotterdam, The Netherlands \\ ${ }^{2}$ Julius Centre for Patient Oriented Research, University Medical Centre Utrecht, Utrecht, The Netherlands \\ ${ }^{3}$ Department of Physiology, Cardiovascular Research Institute Maastricht (CARIM), Maastricht University, Maastricht, \\ The Netherlands \\ ${ }^{4}$ Department of Biophysics, Cardiovascular Research Institute Maastricht (CARIM), Maastricht University, Maastricht, \\ The Netherlands
}

\section{Abstract}

Aims/hypothesis. The insulin resistance syndrome is related to arterial stiffness in diabetic subjects. Whether the insulin resistance syndrome is also related to arterial stiffness in non-diabetic subjects is less clear. We studied the association between variables of the insulin resistance syndrome in relation to arterial distensibility in healthy middle-aged non-diabetic women.

Methods. This study was done in 180 non-diabetic women, aged 43-55, selected from the general population. Arterial distensibility was assessed in the carotid artery. The associations were evaluated using linear regression analyses.

Results. Strong associations were found between arterial distensibility and the variables of the insulin resistance syndrome: body mass index, waist-to-hip ratio, high-density-lipoprotein-cholesterol, triglycerides, glucose, insulin, apolipoprotein A1, plasminogen activator inhibitor-1-antigen and tissue-type plasminogen activator-antigen. After additional adjustment for mean arterial pressure, common carotid arterial distensibility remained associated with body mass index: $\beta$-coefficient (95\% confidence interval) per $\mathrm{kg} / \mathrm{m}^{2}$ : $-0.24(-0.42 ;-0.06)$; waist-to-hip ratio: $-26.62(-40.59 ;-12.65)$ per $\mathrm{m} / \mathrm{m}$; triglycerides: $-1.42(-2.77 ;-0.08)$ per $\mathrm{mmol} / \mathrm{l}$; plasminogen activator inhibitor-1-antigen: $-0.01(-0.02 ;-0.00)$ per $\mathrm{ng} / \mathrm{ml}$ and borderline significant associated with high-density-lipoprotein-cholesterol: $1.93(-0.01 ; 3.87 ; p=0.07)$ per $\mathrm{mmol} / \mathrm{l}$. Clustering of variables of the insulin resistance syndrome was strongly related to decreased arterial distensibility which remained after adjustment for mean arterial pressure. No association was found between arterial distensibility and variables that are not part of the insulin resistance syndrome: total cholesterol, LDL-cholesterol and apolipoprotein B.

Conclusion/interpretation. The results of this study show that variables of the insulin resistance syndrome are associated with decreased arterial distensibility of the common carotid artery in healthy non-diabetic subjects. [Diabetologia (2000) 43: 665-672]

Keywords Insulin resistance, arterial stiffness, healthy subjects, women, population.
Received: 17 November 1999 and in revised form: 24 January 2000

Corresponding author: J. C.M. Witteman, Department of Epidemiology and Biostatistics, Erasmus University Medical School, Dr. Molewaterplein 50, PO Box 1738, 3000 DR Rotterdam, The Netherlands

Abbreviations: MAP, Mean arterial pressure; PAI-1-antigen, plasminogen activator inhibitor-1-antigen; tPA-antigen, tissue-type plasminogen activator-antigen; $\Delta \mathrm{P}$, pulse pressure; $\mathrm{D}$, diameter; $\Delta \mathrm{D}$, change in diameter during cardiac cycle; $\mathrm{DC}$, distensibility coefficient.
Clustering of risk factors for cardiovascular disease (hypertension, obesity, dyslipidaemia), resistance to insulin-stimulated glucose uptake, and cardiovascular disease is known as the insulin resistance syndrome [1]. The insulin resistance syndrome occurs in non-insulin-dependent diabetic patients but also in non-diabetic people with normal glucose tolerance. In glucose-clamp studies in non-obese subjects with normal oral glucose tolerance, resistance to insulin-stimulated glucose uptake was found in approximately $25 \%$ of these subjects [2]. These subjects secrete high 
amounts of insulin to maintain normal glucose concentration despite their insulin resistance, leading to hyperinsulinaemia.

Hyperglycaemic conditions lead to increased arterial stiffness by increased collagen cross-linking due to non-enzymatic glycation [3-5]. One study showed that insulin resistance is associated with decreased arterial distensibility in Type II (non-insulin-dependent) diabetic subjects [6] but little is known about the effect of insulin resistance on arterial distensibility in healthy non-diabetic subjects. As decreased arterial distensibility is associated with increased cardiovascular risk $[7,8]$, it is of importance to know whether insulin resistance is associated with decreased arterial distensibility in healthy subjects.

The Atherosclerosis Risk in Communities (ARIC) study examined the association between fasting glucose and insulin concentrations with arterial stiffness in a large population-based setting and found these variables to be associated with increased arterial stiffness indexes. This study however, did not include all variables of the insulin resistance syndrome [9]. One study described an association of insulin resistance assessed by the euglycaemic hyperinsulinaemic clamp with decreased arterial compliance and distensibility in healthy young subjects, which was more pronounced in women than in men [10]. This study was done in a relatively small group of 17 men and 17 women. The objective of our study was to examine the association between arterial distensibility and all variables of the insulin resistance complex in 180 healthy non-diabetic women. Variables of the insulin resistance complex included BMI, waist-to-hip ratio, HDL-cholesterol, triglycerides, apolipoprotein A1, PAI-1-antigen and tPA-antigen. Additionally, we examined whether clustering of the variables of the insulin resistance syndrome within one subject is related to arterial distensibility.

\section{Subjects and methods}

Study group. Our study group comprised women aged 43-55 years selected from the general population and who participated in a study on the cardiovascular effects of natural menopause. Women were selected from 6845 respondents to a mailed questionnaire about menopause which was sent to all women aged 40 to 60 years living in the town of Zoetermeer, The Netherlands (response rate $54 \%$ ). Exclusion criteria were diabetes mellitus, prevalent clinical cardiovascular disease, and use of antihypertensive or cholesterol-lowering drugs. Women reporting use of female hormones (hormone replacement therapy or oral contraceptives) within 6 months prior to the clinical examination were also excluded, as were women currently smoking five or more cigarettes per day. Eligible for the study were women with an early or late menopause. Of these subjects, 186 pre- and postmenopausal women, aged 43-55 years, were randomly selected. The study was approved by the medical ethics committee of the Erasmus University Medical School. All women gave informed consent.
Clinical and biochemical variables. During an examination at the research centre, a medical history was taken by a physician. Height, weight, and waist and hip circumference were measured with indoor clothes without shoes. BMI (weight in $\mathrm{kg}$ divided by height in $\mathrm{m}^{2}$ ) and waist-to-hip ratio were computed. Alcohol drinking habits and a history of cigarette smoking were obtained by a standardised questionnaire. Blood pressure was assessed with a Dinamap automatic blood pressure recorder (Critikon, Tampa, Fla., USA). After 5 min rest in the supine position, blood pressure was read four times at the right upper arm, and the mean was used in the analyses. Hypertension was defined as a systolic blood pressure of $160 \mathrm{mmHg}$ or higher or a diastolic blood pressure of $95 \mathrm{mmHg}$ or higher or both. Pulse pressure $(\Delta \mathrm{P})$ was defined as systolic blood pressure minus diastolic blood pressure. Mean arterial pressure (MAP) was calculated by the following formula: diastolic blood pressure $+1 / 3$ times the pulse pressure.

Venous blood samples were drawn from each subject after a 12 -h fast. The samples were stored at $-80^{\circ} \mathrm{C}$, and subsequently serum variables were determined using a Kone Specific Analyzer (Kone Instruments, Espoo, Finland). Total cholesterol was measured with an automated enzymatic method [11], using the CHOD-PAP High Performance reagent kit from Boehringer Mannheim (Germany). We measured HDL-cholesterol by the phosphotungstate method according to Burstein [12] with a minor modification as described by Grove [13]. The overall coefficients of variation for total cholesterol and HDL-cholesterol were $2.9 \%$ and $3.7 \%$, respectively. We computed LDL-cholesterol with the Friedewald formula [14]. Triglycerides were determined using a reagent kit from Boehringer Mannheim (Germany) after enzymatic hydrolysis of the triglycerides with subsequent determination of liberated glycerol by colorimetry. No correction was made for serum free glycerol. The overall coefficient of variation of this method did not exceed $3.2 \%$. Apolipoprotein A1 and B were measured by an automated turbidimetric immuno-assay using the reagent kits of Orion Diagnostics (Espoo, Finland). Glucose was enzymatically determined by the hexokinase method (Instruchemie, Hilversum, The Netherlands). Serum insulin was determined by metric assay (Biosource Diagnostics, Fleuris, Belgium). This assay has no cross-reactivity with either pro-insulin or C-peptide. Plasminogeeen activator inhibitor-1 (PAI1)-antigen and tissue-type plasminogen activator (tPA)-antigen were determined by ELISA (Innotest PAI-1, Innogenetics NV, Zwijngaarde, Belgium and Imulyse, Biopool, Umea, Sweden, respectively). Fasting insulin concentrations were used as a measure of insulin resistance [15].

Arterial distensibility. Arterial distensibility was assessed by measuring the distensibility of the common carotid artery and expressed as the distensibility coefficient (DC). The vessel wall motion of the right common carotid artery was measured by means of a Duplex scanner with an operating frequency of 7.5 MHz (Ultramark IV, ATL, Bothell, Wash., USA) connected to a vessel wall movement detector system. The details of this technique have been described elsewhere [16, 17]. Briefly, this system enables the transcutaneous assessment of displacement of the arterial walls during the cardiac cycle and, hence, the time-dependent changes in arterial diameter relative to its diastolic diameter at the start of the cardiac cycle. Subjects were instructed to refrain from smoking and from having coffee, tea, alcohol or pain medication on the day of measurement and from drinking alcohol on the day before. Subjects were placed in a supine position with the head tilted slightly to the contralateral side for the measurements in the common carotid artery. A region at $1.5 \mathrm{~cm}$ proximal to the origin of the bulb of the carotid artery was identified using B-mode ultrasonogra- 
phy. Based on the B-mode recording an M-line perpendicular to the artery was selected and the received radio frequency signals were recorded over five cardiac cycles and digitally stored. The displacement of the arterial walls was obtained by processing the radio frequency signals originating from two selected sample volumes positioned over the anterior and posterior walls. The successive values of the end-diastolic diameter (D), the absolute stroke change in diameter during systole $(\Delta \mathrm{D})$, and the relative stroke change in diameter $((\Delta \mathrm{D}) / \mathrm{D})$ were computed from the recording during five cardiac cycles. Blood pressure was measured with a Dinamap automatic blood pressure recorder and read four times at the right upper arm during the measurement session. The mean was taken as the subject's reading. The cross-sectional arterial wall distensibility coefficient was calculated according to the following equation [18]: $\mathrm{DC}=(2 \Delta \mathrm{D} / \mathrm{D}) / \Delta \mathrm{P}\left(10^{-3} \mathrm{kPa}^{-1}\right)$

With this system a wall displacement of a few micrometers can be resolved [16] and diameter (D), $\Delta \mathrm{D}, \Delta \mathrm{D} / \mathrm{D}, \mathrm{DC}$ can be assessed reliably [17]. The arterial wall properties determined in this way are defined as the relative changes in arterial cross-sectional area, expressed in terms of diameter, for a given change in pressure. They reflect a combination of passive elastic properties and active components induced by smooth muscle cells. All measurements were done by a single examiner. A reproducibility study was carried out in which 15 participants underwent a second examination within 1 month from the initial examination. The coefficients of variation for distension and the lumen diameter were $8.5 \%$ and $1.2 \%$, respectively. In our study, measurements were restricted to the right side to save time. In previous studies no differences could be detected between arterial wall properties of the right and left common carotid artery (unpublished data).

Statistical analysis. Data on distensibility of the common carotid artery were missing for six subjects of the study group of 186 subjects; therefore the analyses were based on 180 subjects. The clinical and biochemical features of the population are presented as mean \pm standard deviation, as median (interquartile range) for variables with a skewed distribution, or as percentages. Pearson's correlation coefficients or Spearman's correlation coefficients in case of variables with a skewed distribution were calculated for the clinical and biochemical variables with MAP. The associations of arterial distensibility with the clinical and biochemical variables were studied using multiple linear regression analyses of the DC on each variable separately, adjusted for age and menopausal status (model A), for age, menopausal status and MAP (model B) and for age, menopausal status, MAP and insulin (model C). Model B was additionally extended with MAP-squared. For variables which were still associated with arterial distensibility of the common carotid artery in model $\mathrm{B}$, we calculated mean values per tertile of the DC adjusted for age, menopausal status and MAP by one way analyses of covariance. A test for trend was done using multiple linear regression analysis with the tertiles of the DC as ordinal variable. To examine whether the strong associations of body composition variables with distensibility were mediated by arterial diameter, we additionally carried out analyses with $(2 \Delta \mathrm{D} / \Delta \mathrm{P})$ instead of the $\mathrm{DC}$ as dependent variable in relation to $\mathrm{BMI}$ and waist-to-hip ratio, adjusted for age, MAP and D.

Finally, we evaluated whether clustering of variables of the insulin resistance syndrome was associated with decreased arterial distensibility. For this purpose, we created a compound score referred to as a clustering score. It was calculated as the sum of Z-scores of the variables of the insulin resistance syndrome (BMI, waist-to-hip ratio, HDL-cholesterol, triglycerides, glucose, insulin, apolipoprotein A1, PAI-1-antigen and
Table 1. Baseline characteristics of 180 women aged 43 to 55 years from the Zoetermeer study population

\begin{tabular}{|c|c|}
\hline Variable & $\begin{array}{l}\text { Subjects } \\
n=180\end{array}$ \\
\hline Age (years) & $50.9 \pm 2.3$ \\
\hline $\mathrm{BMI}\left(\mathrm{kg} / \mathrm{m}^{2}\right)$ & $24.9 \pm 4.0$ \\
\hline Waist-to-hip ratio & $0.77 \pm 0.05$ \\
\hline \multicolumn{2}{|l|}{ Smoking status (\%) } \\
\hline Current ${ }^{\mathrm{a}}$ & 6.5 \\
\hline Past & 40.3 \\
\hline Systolic blood pressure $(\mathrm{mm} \mathrm{Hg})$ & $121 \pm 14$ \\
\hline Diastolic blood pressure ( $\mathrm{mm} \mathrm{Hg}$ ) & $68 \pm 10$ \\
\hline Pulse pressure $(\mathrm{mm} \mathrm{Hg})$ & $52 \pm 10$ \\
\hline Hypertension (\%) & 2.2 \\
\hline Total cholesterol (mmol/l) & $6.2 \pm 1.0$ \\
\hline HDL-cholesterol (mmol/l) & $1.6 \pm 0.4$ \\
\hline LDL-cholesterol (mmol/l) & $4.1 \pm 0.9$ \\
\hline Triglycerides $(\mathrm{mmol} / \mathrm{l})^{\mathrm{b}}$ & $1.0(0.8-1.3)$ \\
\hline Glucose $(\mathrm{mmol} / \mathrm{l})$ & $5.5 \pm 0.6$ \\
\hline Insulin $(\mathrm{pmol} / \mathrm{l})^{\mathrm{b}}$ & $44(32-59)$ \\
\hline Apolipoprotein A1 (mg/dl) & $154 \pm 32$ \\
\hline Apolipoprotein B (mg/ml) & $102 \pm 26$ \\
\hline PAI-1-antigen $(\mathrm{ng} / \mathrm{ml})^{\mathrm{b}}$ & $53(34-85)$ \\
\hline tPA-antigen (ng/ml) & $6.3 \pm 2.4$ \\
\hline Diameter (mm) & $6.7 \pm 0.6$ \\
\hline Distensibility (mm) & $0.38 \pm 0.10$ \\
\hline Distensibility coefficient $\left(10^{-3} \mathrm{kPa}^{-1}\right)$ & $16.7 \pm 5.0$ \\
\hline
\end{tabular}

Data are given as mean \pm standard deviation, as median (interquartile range) for variables with skewed distribution ${ }^{\mathrm{a}}$ or as percentages

${ }^{\mathrm{b}}$ Subjects who smoked 5 or more cigarettes a day were excluded from the study population

tPA-antigen). A Z-score indicates the position of an individual value of a variable in the total distribution of the variable in the population and is calculated as follows: (individual value mean value)/standard deviation. The association between this variable and arterial distensibility was studied using linear regression adjusted for age and MAP. All analyses were done using the BMDP statistical package.

\section{Results}

The clinical and biochemical characteristics of the study group are listed in Table 1 . Table 2 shows the correlation between MAP and different clinical and biochemical variables. All clinical and biochemical variables were highly associated with MAP.

The association between clinical and biochemical variables of the insulin resistance complex and arterial distensibility, adjusted for age and menopausal status (model A) and after additional adjustment for MAP (model B) are shown in Table 3. In model A, associations were found of BMI, waist-to-hip ratio, HDL-cholesterol, triglycerides, glucose, insulin, apolipoprotein A1, PAI-1-antigen, and tPA-antigen with arterial distensibility of the common carotid artery. As expected, BMI, waist-to-hip ratio, triglycerides, glucose, insulin, PAI-1-antigen and tPA-antigen were negatively, and HDL-cholesterol and apolipo- 
Table 2. Correlation of clinical and biochemical variables with mean arterial pressure in 180 women aged 43 to 55 years from the Zoetermeer study population

\begin{tabular}{lcc}
\hline & $r$ & $p$-value \\
\hline BMI $\left(\mathrm{kg} / \mathrm{m}^{2}\right)$ & 0.19 & $<0.001$ \\
Waist-to-hip ratio & 0.19 & $<0.001$ \\
Total cholesterol $(\mathrm{mmol} / \mathrm{l})$ & 0.18 & 0.01 \\
HDL-cholesterol $(\mathrm{mmol} / \mathrm{l})$ & -0.17 & 0.02 \\
LDL-cholesterol $(\mathrm{mmol} / \mathrm{l})$ & 0.17 & 0.02 \\
Triglycerides $(\mathrm{mmol} / \mathrm{l})^{\mathrm{a}}$ & 0.32 & $<0.001$ \\
Glucose $(\mathrm{mmol} / \mathrm{l})_{\text {Insulin }(\mathrm{pmol} / \mathrm{l})^{\mathrm{a}}}$ & 0.31 & $<0.001$ \\
Apolipoprotein A1 (mg/dl) & 0.25 & 0.001 \\
Apolipoprotein B $(\mathrm{mg} / \mathrm{ml})$ & -0.15 & 0.04 \\
PAI-1-antigen $(\mathrm{ng} / \mathrm{ml})^{\mathrm{a}}$ & 0.23 & 0.001 \\
tPA-antigen $(\mathrm{ng} / \mathrm{ml})$ & 0.22 & 0.003 \\
\end{tabular}

Results are presented as Pearson's correlation-coefficients or as Spearman's correlation coefficients for variables with a skewed distribution ${ }^{\mathrm{a}}$ and $p$-values

protein A1 positively, associated with arterial distensibility. We did not find associations of the variables that are not part of the insulin resistance syndrome (total cholesterol, LDL-cholesterol and apolipoprotein B) with common carotid artery distensibility. After additional adjustment for MAP (model B), BMI, waist-to-hip ratio, triglycerides and PAI-1-antigen remained significantly associated with decreased common carotid artery distensibility $(p=0.01$ for BMI, $p<0.001$ for waist-to-hip ratio, $p=0.04$ for triglycerides and $p=0.05$ for PAI-1-antigen). High density lipoprotein cholesterol remained borderline significantly associated $(p=0.07)$ with decreased common carotid artery distensibility. The clustering score had a strong negative association with common carotid artery distensibility, which remained after additional adjustment for MAP. Adding MAP-squared to model $\mathrm{B}$ did not alter the results (data not shown). The per- centage-explained variance of the distensibility coefficient by the various variables of the insulin resistance complex in model $\mathrm{B}$ ranged from $10.8 \%$ (apolipoprotein B) to $18.7 \%$ (waist-to-hip ratio). Figure 1 shows mean values of those parameters of the insulin resistance complex which were still (borderline) associated to decreased arterial distensibility after additional adjustment for MAP (model B) per tertile of arterial distensibility of the common carotid artery adjusted for age, menopausal status and MAP. As expected, the highest values BMI, waist-to-hip ratio, triglycerides, and PAI-1-antigen and the lowest value of HDL cholesterol were found in the lowest tertile of the DC. In all cases except for PAI-1-antigen, the test for trend was significant ( $p=0.003$ for BMI and waist-to-hip ratio, 0.009 for HDL-cholesterol, and 0.038 for triglycerides). In model $\mathrm{C}$, with further adjustment for insulin, no attenuation of the associations of BMI and waist-to-hip ratio with common carotid arterial distensibility was found $[\beta$-coefficient (95\% confidence interval) per $\mathrm{kg} / \mathrm{m}^{2}$ increase for BMI: $-0.24(-0.44 ;-0.04)$ and per unit increase for waist-to-hip ratio: $-25.6(-40.0 ;-11.3)]$. The associations of triglycerides and PAI-1-antigen with common carotid artery distensibility was lost after additional adjustment for insulin [ $\beta$-coefficient (95\% confidence interval) per $\mathrm{mmol} / \mathrm{l}$ increase for triglycerides: $-1.31(-2.80 ; 0.17)$, per $\mathrm{ng} / \mathrm{ml}$ increase for PAI-1-antigen: $-0.01(-0.02 ; 0.002)]$.

We next carried out analyses with $(2 \Delta \mathrm{D} / \Delta \mathrm{P})$ index as dependent variable in relation to $\mathrm{BMI}$ and waistto-hip ratio. Waist-to-hip ratio was strongly associated to the $(2 \Delta \mathrm{D} / \Delta \mathrm{P})$ index, adjusted for age, MAP and $\mathrm{D}$ : $\beta$-coefficient ( $95 \%$ confidence interval) for waist-to-hip ratio: $-10.3(-17.28 ;-3.30)$, whereas BMI was not associated with the $(2 \Delta \mathrm{D} / \Delta \mathrm{P})$ index: $\beta$ coefficient $\left(95 \%\right.$ confidence interval) per $\mathrm{kg} / \mathrm{m}^{2}$ : $-0.06(-0.15 ; 0.03)$.

Table 3. Association of clinical and biochemical variables with the distensibility coefficient of the common carotid artery in 180 women aged 43 to 55 years from the Zoetermeer study population

\begin{tabular}{|c|c|c|c|c|}
\hline & \multicolumn{2}{|l|}{ Model A } & \multicolumn{2}{|l|}{ Model B } \\
\hline & $\beta$-coefficient & $95 \%$ C.I. & $\beta$-coefficient & $95 \%$ C.I. \\
\hline $\mathrm{BMI}\left(\mathrm{kg} / \mathrm{m}^{2}\right)$ & -0.34 & -0.52 to -0.16 & -0.24 & -0.42 to -0.06 \\
\hline Total cholesterol (mmol/l) & -0.01 & -0.77 to 0.80 & 0.27 & -0.48 to 1.03 \\
\hline HDL-cholesterol (mmol/l) & 2.63 & 0.66 to 4.61 & 1.93 & -0.01 to 3.87 \\
\hline LDL-cholesterol (mmol/l) & -0.03 & -0.87 to 0.81 & 0.22 & -0.60 to 1.03 \\
\hline Triglycerides $(\mathrm{mmol} / \mathrm{l})$ & -2.23 & -3.51 to -0.95 & -1.42 & -2.77 to -0.08 \\
\hline Apolipoprotein A1 (mg/dl) & 0.03 & 0.002 to 0.05 & 0.02 & -0.01 to 0.04 \\
\hline Apolipoprotein B (mg/ml) & -0.02 & -0.05 to 0.009 & -0.01 & -0.04 to 0.02 \\
\hline PAI-1-antigen (ng/ml) & -0.02 & -0.03 to -0.006 & -0.01 & -0.02 to -0.00 \\
\hline tPA-antigen $(\mathrm{ng} / \mathrm{ml})$ & -0.33 & -0.63 to -0.03 & -0.18 & -0.48 to 0.11 \\
\hline Clustering score & -0.27 & -0.38 to -0.15 & -0.20 & -0.32 to -0.07 \\
\hline
\end{tabular}

Results are presented as $\beta$-coefficients (increase in carotid distensibility in $10^{-3} \mathrm{kPa}^{-1}$ per unit increase of the parameter) and $95 \%$ C.I. (confidence interval). Model A is adjusted for age and menopausal status. Model B is adjusted for age, menopausal status and mean arterial pressure 

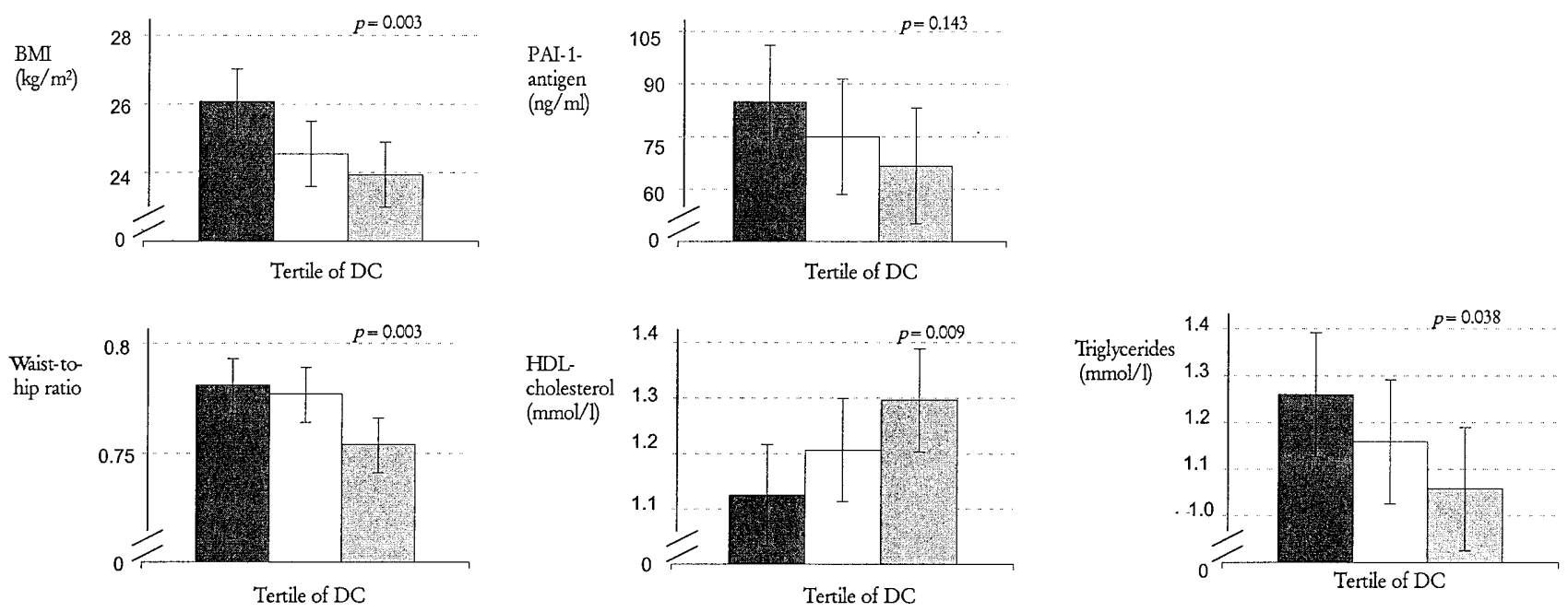

Fig. 1. Estimated marginal means ( $95 \%$ confidence intervals) of various variables of the insulin resistance complex per tertile of distensibility coefficient, adjusted age, menopausal status and mean arterial blood pressure. $\square 1^{\text {st }}$ tertile $\square 2^{\text {nd }}$ tertile $3^{\text {rd }}$ tertile $p$ values indicates $p$ for trend

\section{Discussion}

The results of our study indicate that in healthy nondiabetic middle-aged women variables of the insulin resistance syndrome are strongly associated with reduced distensibility of the common carotid artery. Furthermore, common carotid artery distensibility had a strong inverse association with clustering of variables of the insulin resistance syndrome in one subject. Variables which are not part of the insulin resistance syndrome (total cholesterol, LDL-cholesterol and apolipoprotein B) were not related to arterial distensibility.

Some methodological issues need to be discussed before we can interpret our findings. Firstly, by calculating the distensibility coefficient, distension of the common carotid artery is adjusted for pulse pressure measured in the brachial artery. We thereby assume that pulse pressure measured in the brachial artery is representative of pulse pressure in carotid arteries. In dogs, it has been shown that pulse pressure in the brachial artery is linearly related to blood pressure in the carotid artery over a wide range of blood pressures [19]. It is known, however, that the arterial pressure waves undergo transformation in the arterial tree and therefore the pulse pressure is higher in the brachial artery than in more central vessels like the carotid artery [20]. On the other hand, non-invasive cuffbased measurement of blood pressure underestimates pulse pressure [21]. Several groups showed the validity of the use of brachial pressures [22-24]. Secondly, in analyses with arterial distensibility, a measure very dependent on blood pressure, adequate correction for blood pressure is of the utmost importance. The distensibility coefficient is calculated by dividing the relative distension by the pulse pressure. Despite this correction, the distensibility coefficient has a strong negative association with MAP. A higher MAP in the artery stretches the elastin and collagen fibres in the arterial wall, making the artery less distensible. Blood pressure is one of the major determinants of arterial stiffness and also part of the insulin resistance syndrome. Therefore, we repeated the analyses after additional adjustment for MAP. This attenuated the associations, which in part could be due to over-correction because arterial stiffness is not only a consequence of high blood pressure but will by itself also result in a higher blood pressure. After adjustment for MAP, the variables BMI, waist-to-hip ratio, triglycerides and PAI-1 antigen remained associated and HDL-cholesterol borderline-associated with decreased arterial distensibility. Clustering of the variables of the insulin resistance syndrome remained strongly associated with a considerable decrease of common carotid artery distensibility after additional adjustment for MAP. On the other hand, adjustment for MAP in a linear model might not be adequate as the relation between arterial distension and MAP flattens off at higher blood pressures, making the overall relation non-linear. This could result in residual confounding. Although, in the normal pressure range, as in this study, the flattening-off barely occurs in elastic arteries as the common carotid artery, we additionally adjusted the models for MAP-squared. This term was not associated with arterial distensibility in any of the models and did not alter the results. Table 2 shows that MAP is related not only to the variables of the insulin resistance syndrome but also has a strong relation with total cholesterol, LDL cholesterol and apolipoprotein B. The absence of an association between these biochemical variables and arterial distensibility (Table 3) makes it unlikely that the observed associations can be explained solely by residual confounding by blood pressures. 
Our study is part of a study designed to evaluate the effect of natural menopause. This study includes both premenopausal and postmenopausal women. As hormonal status is known to affect variables of the insulin resistance complex [25] and hormonal status might also have an effect on arterial distensibility [26] hormonal status could act as a confounder in the association under study. We therefore adjusted all the models for menopausal status.

The insulin resistance syndrome is associated with arterial stiffness in non-insulin-dependent diabetic patients [6]. There are few reports concerning the association of insulin resistance syndrome with arterial stiffness in normal non-diabetic people. Results from a population-based study suggest that insulin concentrations affect arterial stiffness independently of their effects on atherosclerosis [9]. Some studies examined serum lipids, lipoprotein concentrations and serum glucose and insulin as determinants of arterial stiffness in various populations. These studies, however, report conflicting results. Some studies found no association between lipids and arterial distensibility [27-29]. Some studies found no association between apolipoprotein A1 and apolipoprotein B with arterial stiffness after adjustment for blood pressure [29, 30]. Several studies did find an association between serum lipids and arterial distensibility, sometimes this association was positive whereas most studies found a negative association [30-34]. Serum glucose or insulin or both have been found to be negatively associated with arterial distensibility in several studies [9, 29-31,33] but some of these studies did not adequately adjust for blood pressure. One study found no correlation between area under the glucose tolerance curve and arterial distensibility after adjustment for age and blood pressure [34]. There are several possible explanations for conflicting results in these previous studies. Firstly, most of these studies were done in small groups of subjects. Secondly, the different studies were done on heterogeneous study populations, ranging from healthy cohorts to hypertensive, hypercholesterolaemic or diabetic patients. Thirdly, there is a large variation in measures of arterial distensibility. It is still not clear whether locally measured arterial distensibility represents the same arterial wall characteristics as pulse wave velocity which determines arterial stiffness measured over a large part of the arterial tree. Furthermore, some studies presented results that were not adjusted for age or blood pressure. Finally, contradictory findings with respect to the direction of the association between arterial distensibility and serum lipids can be due to differences in age of the study populations. There is compelling evidence that early nonsclerotic atheromatous changes of the arterial wall in relatively young subjects (age $<50$ years) decrease rather than increase arterial stiffness [35-37]. One study concerned the association of insulin resistance with arte- rial stiffness in healthy subjects and found this association to be more pronounced in women than in men [10]. This association was independent of MAP, lipid concentrations and body composition.

The association of insulin with arterial distensibility was lost after adjustment for MAP. This is in contrast with another study [10] in which an association was found of insulin resistance, assessed by the euglycaemic, hyperinsulinaemic clamp, with arterial distensibility independent of MAP. We used fasting insulin as a marker of insulin resistance although it is known that fasting insulin is a reasonable albeit imperfect marker of insulin resistance in subjects with normal glucose tolerance [15].

Although BMI and waist-to-hip ratio are strongly related to insulin [38], the association of BMI and waist-to-hip ratio with arterial distensibility remained after further adjustment for insulin (model C). The associations of HDL-cholesterol, triglycerides and PAI-1-antigen with arterial distensibility did not remain after additional adjustment for insulin. Some authors suggested that obesity could lead to an increased arterial diameter and therefore carried out analyses in which they used the $(\Delta \mathrm{D} / \Delta \mathrm{P})$ index as a dependent variable [10]. They found no association of this index with variables of body composition and concluded that only arterial diameter, and not arterial distensibility, is related to variables of body composition. In our study, in agreement with the other study, $B M I$ also was not associated with the $(2 \Delta \mathrm{D} / \Delta \mathrm{P})$ index, independent of arterial diameter. Waist-to-hip ratio, however, had a strong inverse association with the $(2 \Delta \mathrm{D} / \Delta \mathrm{P})$ index, independent of age, MAP and arterial diameter.

Hyperglycaemic conditions can lead to increased arterial stiffness by increased collagen cross-linking due to non-enzymatic glycation [3-5]. This could be the mechanism underlying our observation of an association between the insulin resistance syndrome and decreased arterial distensibility. Arterial stiffness plays a part in the aetiology of isolated systolic hypertension and increased cardiac load [39] and has been found to be associated with increased cardiovascular risk $[7,8]$. Therefore, knowledge of potential modifying determinants of arterial stiffness is possibly important in the prevention of cardiovascular disease. Recent evidence suggests that there is therapeutic potential to treat arterial stiffness induced by hyperglycaemia by breakers of advanced glycation end products [40].

In summary, the results of our study show that variables of the insulin resistance syndrome are associated with decreased arterial distensibility in healthy women.

Acknowledgements. This study was supported by a grant from the Health Research and Development Council, The Hague, The Netherlands (grant no. 2827210 to Dr J.C.M. Witteman) 
and by a grant from the Netherlands Hearts-Foundation. The Hague, The Netherlands (grant no. 92381 to Dr J.C. M. Witteman). We thank J.M. Vergeer-Drop for carrying out the laboratory measurements and T. Stehmann and I. T. M. Haumersen for data collection.

\section{References}

1. DeFronzo RA, Ferrannini E (1991) Insulin resistance. A multifaceted syndrome responsible for NIDDM, obesity, hypertension, dyslipidemia, and atherosclerotic cardiovascular disease. Diabetes Care 14: 173-194

2. Reaven GM (1988) Banting lecture 1988. Role of insulin resistance in human disease. Diabetes 37: 1595-1607

3. Winlove CP, Parker KH, Avery NC, Bailey AJ (1996) Interactions of elastin and aorta with sugars in vitro and their effects on biochemical and physical properties. Diabetologia 39: 1131-1139

4. Airaksinen KE, Salmela PI, Linnaluoto MK, Ikaheimo MJ, Ahola K, Ryhanen LJ (1993) Diminished arterial elasticity in diabetes: association with fluorescent advanced glycosylation end products in collagen. Cardiovasc Res 27: 942-945

5. Meng J, Sakata N, Takebayashi S et al. (1996) Advanced glycation end products of the Maillard reaction in aortic pepsin-insoluble and pepsin-soluble collagen from diabetic rats. Diabetes 45: 1037-1043

6. Emoto M, Nishizawa Y, Kawagishi T et al. (1998) Stiffness indexes beta of the common carotid and femoral arteries are associated with insulin resistance in NIDDM. Diabetes Care 21: 1178-1182

7. Hirai T, Sasayama S, Kawasaki T, Yagi S (1989) Stiffness of systemic arteries in patients with myocardial infarction. A noninvasive method to predict severity of coronary atherosclerosis. Circulation 80: 78-86

8. Lehmann ED, Hopkins KD, Jones RL, Rudd AG, Gosling RG (1995) Aortic distensibility in patients with cerebrovascular disease. Clin Sci (Colch) 89: 247-253

9. Salomaa V, Riley W, Kark JD, Nardo C, Folsom AR (1995) Non-insulin-dependent diabetes mellitus and fasting glucose and insulin concentrations are associated with arterial stiffness indexes. The ARIC Study. Atherosclerosis Risk in Communities Study. Circulation 91: 1432-1443

10. Giltay EJ, Lambert J, Elbers JM, Gooren LJ, Asscheman H, Stehouwer CD (1999) Arterial compliance and distensibility are modulated by body composition in both men and women but by insulin sensitivity only in women. Diabetologia 42: 214-221

11. van Gent CM, van der Voort HA, de Bruyn AM, Klein F (1977) Cholesterol determinations. A comparative study of methods with special reference to enzymatic procedures. Clin Chim Acta 75: 243-251

12. Burstein M, Scholnick HR, Morfin R (1970) Rapid method for the isolation of lipoproteins from human serum by precipitation with polyanions. J Lipid Res 11: 583-595

13. Grove TH (1979) Effect of reagent $\mathrm{pH}$ on determination of high-density lipoprotein cholesterol by precipitation with sodium phosphotungstate-magnesium. Clin Chem 25: $560-564$

14. Friedewald WT, Levy RI, Fredrickson DS (1972) Estimation of the concentration of low-density lipoprotein cholesterol in plasma, without use of the preparative ultracentrifuge. Clin Chem 18: 499-502

15. Laakso M (1993) How good a marker is insulin level for insulin resistance? Am J Epidemiol 137: 959-965
16. Hoeks AP, Brands PJ, Smeets FA, Reneman RS (1990) Assessment of the distensibility of superficial arteries. Ultrasound Med Biol 16: 121-128

17. Kool MJ, van Merode T, Reneman RS, Hoeks AP, Struyker Boudier HA, Van Bortel LM (1994) Evaluation of reproducibility of a vessel wall movement detector system for assessment of large artery properties. Cardiovasc Res 28: 610-614

18. Reneman RS, van Merode T, Hick P, Muytjens AM, Hoeks AP (1986) Age-related changes in carotid artery wall properties in men. Ultrasound Med Biol 12: 465-471

19. Reneman RS, van Merode T, Brands PJ, Hoeks AP (1992) Inhomogeneities in arterial wall properties under normal and pathological conditions. J Hypertens Suppl 10: S35-S39

20. Nichols WW, O'Rourke MF (1990) McDonald's blood flow in arteries, 3rd edn. Edward Arnold, London

21. Bos WJ, van Goudoever J, Wesseling KH et al. (1992) Pseudohypertension and the measurement of blood pressure. Hypertension 20: 26-31

22. Stefanadis C, Stratos C, Boudoulas H, Kourouklis C, Toutouzas P (1990) Distensibility of the ascending aorta: comparison of invasive and non-invasive techniques in healthy men and in men with coronary artery disease. Eur Heart J 11: 990-996

23. Imura T, Yamamoto K, Kanamori K, Mikami T, Yasuda $H$ (1986) Non-invasive ultrasonic measurement of the elastic properties of the human abdominal aorta. Cardiovasc Res 20: 208-214

24. Stefadouros MA, Dougherty MJ, Grossman W, Craige E (1973) Determination of systemic vascular resistance by a noninvasive technic. Circulation 47: 101-107

25. Spencer CP, Godsland IF, Stevenson JC (1997) Is there a menopausal metabolic syndrome? Gynecological Endocrinology 11: 341-355

26. Westendorp IC, Bots ML, Grobbee DE et al. (1999) Menopausal status and distensibility of the common carotid artery. Arterioscler Thromb Vasc Biol 19: 713-717

27. Barenbrock M, Spieker C, Kerber S et al. (1995) Different effects of hypertension, atherosclerosis and hyperlipidaemia on arterial distensibility. J Hypertens 13: 1712-1717

28. Asmar R, Benetos A, Topouchian J et al. (1995) Assessment of arterial distensibility by automatic pulse wave velocity measurement. Validation and clinical application studies. Hypertension 26: 485-490

29. Taquet A, Bonithon-Kopp C, Simon A et al. (1993) Relations of cardiovascular risk factors to aortic pulse wave velocity in asymptomatic middle-aged women. Eur J Epidemiol 9: 298-306

30. Jonason T, Henrikssen E, Kangro T, Nilsson H, Vessby B, Ringqvist I (1997) Stiffness of the common carotid artery in healthy 50-year-old subjects. Clin Physiol 17: 569-577

31. Kupari M, Hekali P, Keto P, Poutanen VP, Tikkanen MJ, Standerstkjold-Nordenstam CG (1994) Relation of aortic stiffness to factors modifying the risk of atherosclerosis in healthy people. Arterioscler Thromb 14: 386-394

32. Levenson J, Del Pino M, Razavian M, Merli I, Filitti V, Simon A (1992) Hypercholesterolaemia alters arterial and blood factors related to atherosclerosis in hypertension. Atherosclerosis 95: 171-179

33. Neutel JM, Smith DH, Graettinger WF, Weber MA (1992) Dependency of arterial compliance on circulating neuroendocrine and metabolic factors in normal subjects. Am J Cardiol 69: 1340-1344

34. Relf IR, Lo CS, Myers KA, Wahlqvist ML (1986) Risk factors for changes in aorto-iliac arterial compliance in healthy men. Arteriosclerosis 6: 105-108 
35. Blankenhorn DH, Kramsch DM (1989) Reversal of atherosis and sclerosis. The two components of atherosclerosis. Circulation 79: 1-7

36. Lehmann ED, Watts GF, Fatemi-Langroudi B, Gosling RG (1992) Aortic compliance in young patients with heterozygous familial hypercholesterolaemia. Clin Sci (Colch) 83: 717-721

37. Lehmann ED, Hopkins KD, Gosling RG (1993) Aortic compliance measurements using Doppler ultrasound: in vivo biochemical correlates. Ultrasound Med Biol 19: $683-710$
38. Temelkova-Kurktschiev T, Koehler C, Schaper F et al. (1998) Relationship between fasting plasma glucose, atherosclerosis risk factors and carotid intima media thickness in non-diabetic individuals. Diabetologia 41: 706-712

39. Hilden T (1991) The influence of arterial compliance on diastolic blood pressure and its relation to cardiovascular events. J Hum Hypertens 5: 131-135

40. Wolffenbuttel BH, Boulanger CM, Crijns FR et al. (1998) Breakers of advanced glycation end products restore large artery properties in experimental diabetes. Proc Natl Acad Sci USA 95: 4630-4634 